NBSIR 82-2548

\title{
Design Considerations for the Cross-Bridge Sheet Resistor
}

U.S. DEPARTMENT OF COMMERCE

National Bureau of Standards

National Engineering Laboratory

Center for Electronics and Electrical Engineering

Washington, DC 20234

July 1982

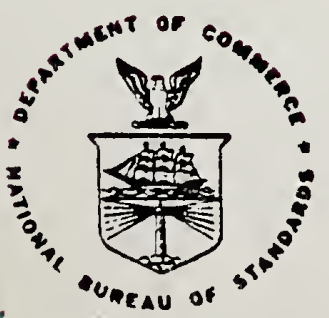



NBSIR $82-2548$

DESIGN CONSIDERATIONS FOR THE CROSS-BRIDGE SHEET RESISTOR

G. P. Carver, R. L. Mattis, and M. G. Buehler

\section{U.S. DEPARTMENT OF COMMERCE}

National Bureau of Standards

National Engineering Laboratory

Center for Electronics and Electrical Engineering

Washington, DC 20234

July 1982

U.S. DEPARTMENT OF COMMERCE, Malcolm Baldrige, Secretary NATIONAL BUREAU OF STANDARDS, Emest Ambler, Director 

Table of Contents

Page

1. Introduction . . . . . . . . . . . . . . . . . . . 1

2. Test Structure Design, Fabrication, and Measurement . . . . . . . 2

3. Symetry Tabs . . . . . . . . . . . . . . . . . 6

4. Shunting Effect Caused by the Voltage Taps . . . . . . . . . . . 8

5. Tap-to-Corner Distance Study . . . . . . . . . . . . . . . 12

6. Summary and Design Considerations . . . . . . . . . . . . . 13

Acknowledgments . . . . . . . . . . . . . . . . . . 16

References . . . . . . . . . . . . . . . . . . 18

List of Figures

1. Line drawings of the cross-bridge sheet resistor without symmetry tabs and the cross bridge with symmetry tabs on test pattern

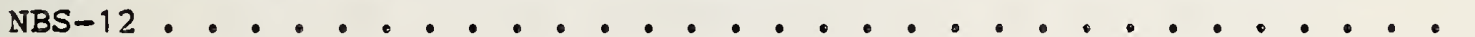

2. Bridge sheet resistor test structures with multiple voltage taps of different widths .......................... 3

3. The bridge tap-to-corner distance study sheet resistors . . . . . . 4

4. Schematic diagram of the bridge . . . . . . . . . . . . . . . 9

5. Outline drawing of a cross-bridge sheet resistor test structure • • 17

List of Tables

1. Average Measured Values (and Standard Deviations) for Metal and Base Layer Devices on Each Wafer for the Sheet Resistance, Asymmetry Factor $F_{A}$, Zero-offset Factor $F_{O}$, Linearity Factor $F_{I}$, and Linewidth for the Nominally 12-1m Linewidth Cross Bridge with Symmetry Tabs .

2. Comparison of Sheet Resistances (and Standard Deviations Expressed as a Percent) Determined Measuring Cross-Bridge Sheet Resistors With and Wi thout Symmetry Tabs . . . . . . . . . . . . . . . .

3. Average Channel Widths Measured Using the Tap-to-Corner Distance Test Structures . . . . . . . . . . . . . . . . . . . 14 


$$
\text { . }
$$


Design Considerations for the Cross-Bridge Sheet Resistor

by

G. P. Carver, R. L. Mattis, and M. G. Buehler*

Center for Electronics and Electrical Engineering

National Bureau of Standards

washington, DC 20234

The cross-bridge sheet resistor test structure is used to obtain

the sheet resistance and electrical linewidth of a conducting lay-

er. It has been used to characterize various conducting layers

found in an integrated circuit fabrication process and to evaluate lithographic equipment used for processing photomasks and wafers. Three geometrical design factors for the cross bridge have been investigated and are shown to cause systematic inaccuracies of less than 1 percent in the sheet resistance and linewidth measurements. Based upon experimental results from sequences of devices with incrementally different geometrical parameters, several design criteria for the cross-bridge sheet resistor have been established.

Key words: cross-bridge structure; linewidth; microelectronic test structure; process control; sheet resistance; test structure.

\section{INTRODUCTION}

The cross-bridge sheet resistor is a microelectronic test structure useful for the automated electrical measurement of the sheet resistance and of the linewidth of conducting layers [1]. Data obtained using this test structure have been used to evaluate integrated circuit process-related sheet resistance spatial nonuniformities [2] and lithography-related linewidth variations [3]. Such specialized applications require a sensitive, precise measurement vehicle which is able to resolve reliably small differences in sheet resistance and linewidth. The design of the cross-bridge sheet resistor and the four-point Kelvin measurement technique associated with it provide a precise measurement tool which can be used, for example, to investigate optical systems using electrical test methods [3]. Automated electrical measurements are important in these types of measurements because statistically significant results are obtained easily and inexpensively [4].

In a previous study, the effect of the geometrical design of a cross-shaped van der Rauw sheet resistor on the accuracy of the measured sheet resistance was determined analytically [5]. The theoretical results of that study were verified experimentally in a succeeding study [6], which also included an investigation of the effects of interferences, such as surface leakage current and joule heating, which affect the accuracy of the results. Several design suggestions which can help minimize interfering effects were given [6]. The advantages of the combined van der Pauw and bridge sheet resistor test structure, called the cross-bridge sheet resistor, are described in a

* Present address: Jet Propulsion Laboratory, Pasadena, CA 91103 
report [1] which also includes experimental results that illustrate the utility of electrical linewidth measurements for process control.

Additional design considerations for the cross-bridge sheet resistor are addressed in the present work. The analysis is impeded by the difficulty of separating random errors which result from process variations from the systematic errors being investigated. Nevertheless, it appears possible to deduce certain information from the measurement results obtained in this study. This information leads to design criteria for cross-bridge test structures which have geometry-imposed systematic errors of 1 percent or less and provides insight into factors which may limit the usable precision of the measurements.

The geometrical design factors discussed in this report are the "symmetry tabs" [1], the voltage tap width, and the bridge voltage tap-to-corner distance.

\section{TEST STRUCTURE DESIGN, FABRICATION, AND MEASUREMENT}

The test structures used in this study were contained in the developmental test pattern NBS-12. This test pattern [7] was assembled to evaluate certain geometrical aspects of the design of the cross-bridge sheet resistor. The test structures primarily intended.for the cross-bridge sheet resistor study include van der Pauw cross sheet resistors, cross-bridge sheet resistors with and without symmetry tabs, bridge sheet resistors with different voltage tab widths, and bridge sheet resistors with different tap positions relative to the end of the conducting channel which forms the bridge. The cross bridge sheet resistors in NBS-12 occur in a sequence of channel widths, $6,12,18$, and $24 \mu \mathrm{m}$. In each of four such sequences the conducting channel of the structures is formed of either the metal, the base (source/drain), the emitter (channel stop) within a base region, or the base-under-emitter layers. One entire series, the base layer, is shown in outline in figures 1 to 3 . In this paper, results are reported on the metal and base layers.

The test pattern was fabricated using a bipolar process [8]. The substrates were 50-mm diameter $\langle 111\rangle$ oriented silicon wafers. Both $p$ - and n-type wafers with room temperature resistivity of approximately $5 \Omega \cdot \mathrm{cm}$ were used. In both cases, the junction depth for the base diffusions was approximately 1.7 .

From the cross bridge sheet resistor, values for the sheet resistance $R_{S}$ and $i$ ts asymetry factor $F_{A^{\prime}}$, offset factor $F_{O}$, and linearity factor $F_{L^{\prime}}$ and the effective linewidth $W_{\text {meas }}$ were determined using previously described procedures and expressions defined in reference 6 . To illustrate the range of values obtained for the above parameters, the results from the cross-bridge structure with symmetry tabs and a nominally 12-lm wide channel are given in table 1 for each of the metal and base layers on two wafers. The value of the current used for each measurement was automatically adjusted to provide a measured voltage of approximately $10 \mathrm{mV}$. For the determination of the linearity factor $F_{L}$, the current was increased to a value twice as large as was used initially for the base layer sheet resistors. (However, the current was halved in the case of the metal layer sheet resistors to avoid attempting to exceed the maximum current available from the constant current supply.) 

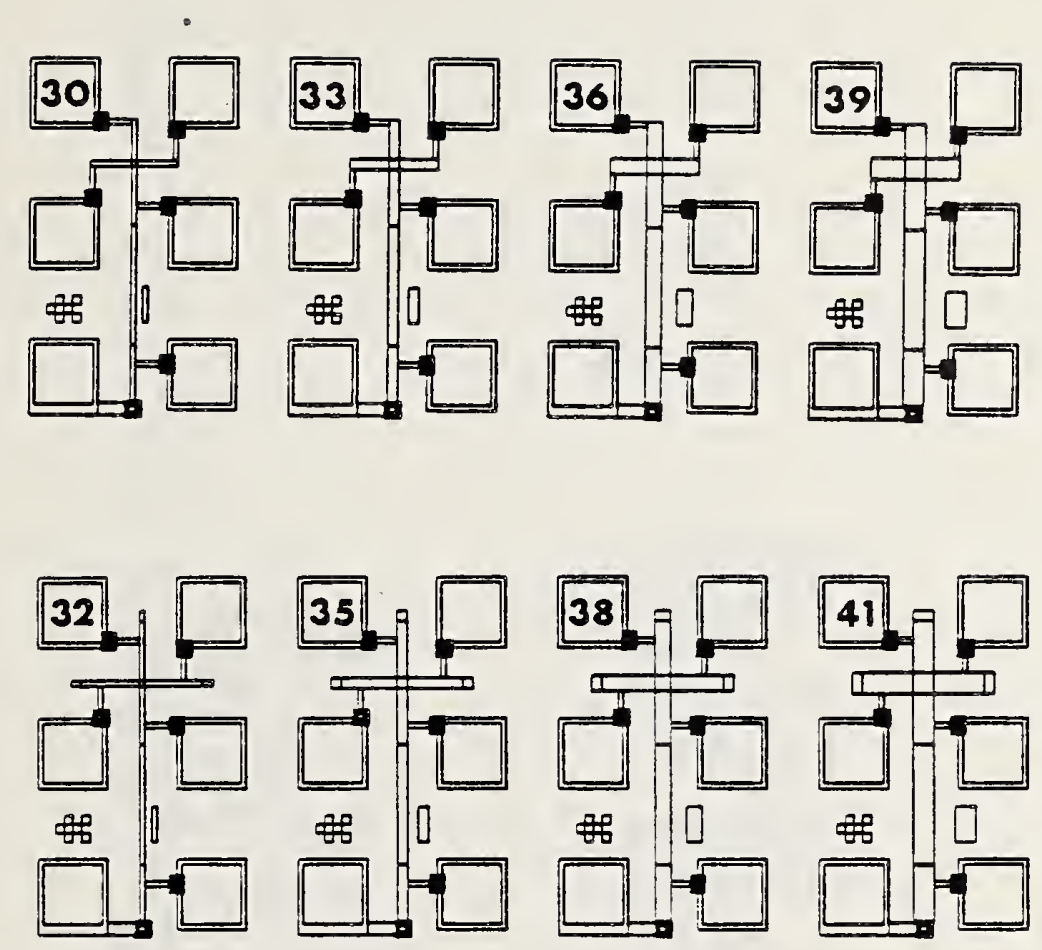

Figure 1. Line drawings of the cross-bridge sheet resistor without symmetry tabs and the cross bridge with symmetry tabs on test pattern NBS-12. These - devices are repeated for the other conducting layers in the process. From left to right, the conducting channel linewidths were designed to be 6,12 , 18 , and $24 \mu \mathrm{m}$.
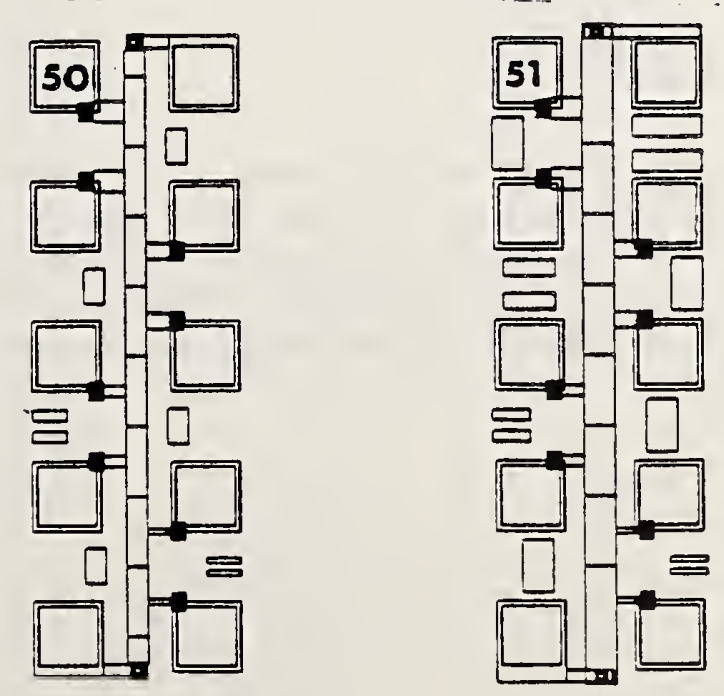

Figure 2. Bridge sheet resistor test structures with multiple voltage taps of different widths. There are four pairs of voltage taps with widths of 6 , 12,18 , and $24 \mathrm{~mm}$ and centerline-to-centerline spacing of $80 \mathrm{~lm}$. The bridge channel is nominally $24 \mathrm{~m}$ wide in one structure and $36 \mathrm{~mm}$ wide in the other. These structures were fabricated in the metal and base diffusion layers. 

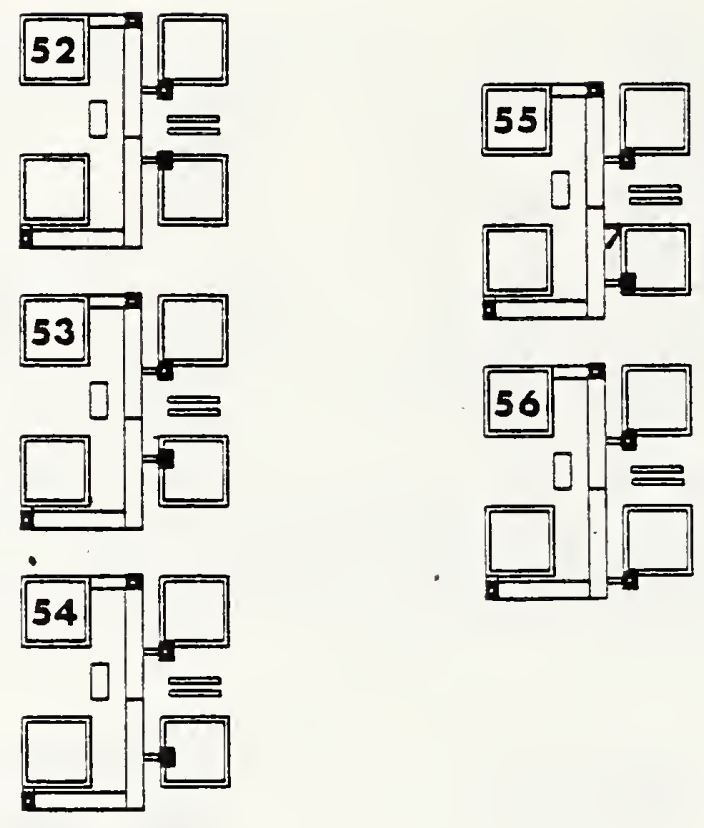

Figure 3. The bridge tap-to-corner distance study sheet resistors. Each structure is a bridge sheet resistor $20 \mu \mathrm{m}$ wide with $6-u$ wide voltage taps. The currant and upper voltage taps are fixed, but the lower voltage tap varies from 80 to $0 \mu \mathrm{m}$, in 20- $\mu \mathrm{m}$ increments, from structure to structure. These structures were fabricated in the metal and base diffusion layers. 


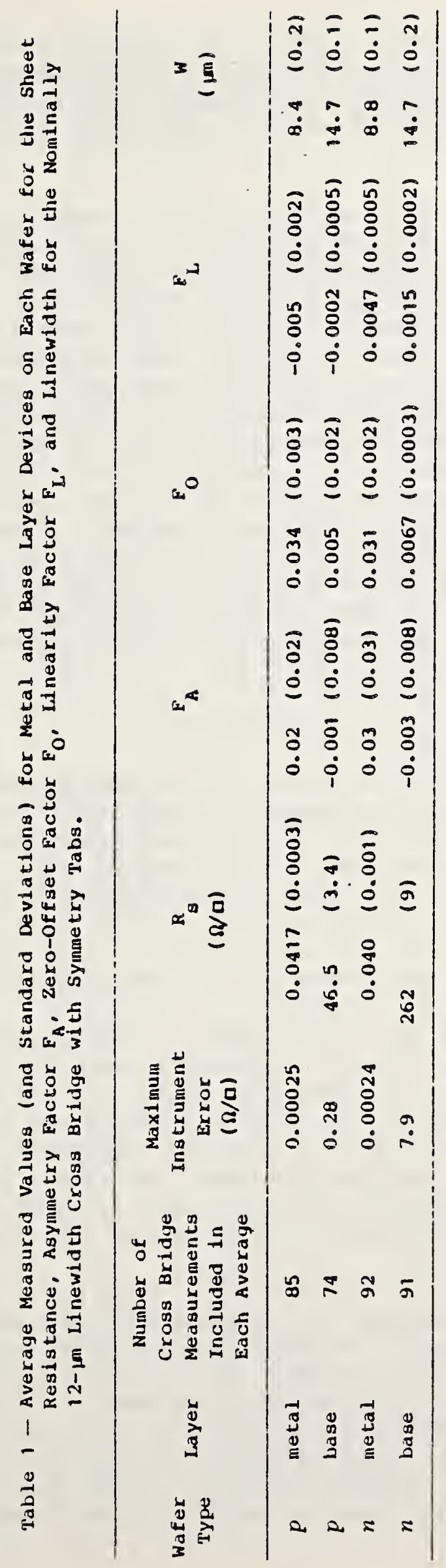


The sheet resistance asymmetry, offset, and linearity factors were used as screening parameters to eliminate outiiers. In this study, data outside three sample standard deviations from the mean value of the sample population were eliminated from the data set which included all the cross bridges of a single geometry.

All measurements were made at room temperature. Temperature variations during collection of a set of data were controlled to within about $1^{\circ} \mathrm{C}$. The data were obtained using a computer-controlled data acquisition system composed of an automated wafer prober and programmable scanners, constant current supply, and digital voltmeter. On their most sensitive scales, the current source and the five-digit voltmeter have microampere and microvolt resolution, respectively. Based on the manufacturer's published specified maximum inaccuracy of the instruments, including the basic instrument uncertainty, the inaccuracy caused by temperature and line voltage variations, and the inaccuracy caused by "drift," the expected uncertainties were $\pm 6 \mu \mathrm{A}+$ 0.01 percent of the programmed value for the constant current source and \pm 5 $\mu V+0.005$ percent of the reading for the voltmeter. (These values are estimated for the operating conditions of the instruments during the measurements.) As listed in table 1, the maximum instrument errors in measuring the sheet resistance derived from these considerations are much smaller than the sample standard deviations observed for the measured average sheet resistance values.

\section{SYMMETRY TABS}

Symmetry tabs on the cross-bridge sheet resistor are extensions past the taps of three arms of the cross to compensate for any effect the bridge, which is an extension to the fourth arm, may have on the measurement of the sheet resistance $R_{S}[1]$. The symmetry tabs are included on the structures shown in the bottom row of figure 1. The cross-bridge dimensions are: bridge length L, $180 \mathrm{\mu m}$; conducting channel width $\mathrm{W}, 6$ to $24 \mathrm{\mu m}$ in 6 - $\mathrm{m}$ increments; voltage tap width M, $6 \mathrm{um}$; minimum "tap-to-corner" distance, $39 \mathrm{um}$; and tap length $\mathrm{N}$, 18 to $24 \mathrm{~m}$ (see fig. 4). The length of the symmetry tabs is $34 \mathrm{~m}$.

Within the uncertainties of this experiment, no significant difference was observed between measurements on the structures without symmetry tabs and the structures with the extensions. This conclusion is based upon data summarized in table 2. The column at the far right shows the mean values of the relative difference between the sheet resistance measured, first using the cross-bridge resistor with tabs and the sheet resistance measured, then using the structure wi thout symmetry tabs in each chip for each linewidth. The differences appeared to be randomly distributed in amplitude and in spatial position on the wafer.

As seen in the right-hand column, the relative differences are smaller than 1 percent, except in one case. In every case, however, these differences are smaller than the uncertainties (standard deviations) in the mean values of the measured resistivities. This latter relationship is not sufficient to guarantee that the differences are statistically insignificant because there are systematic variations in sheet resistance across the wafers. Systematic variations in $R_{S}$ cause the standard deviation of the mean sheet resistance values to be larger than they would be solely in the presence of randomly 


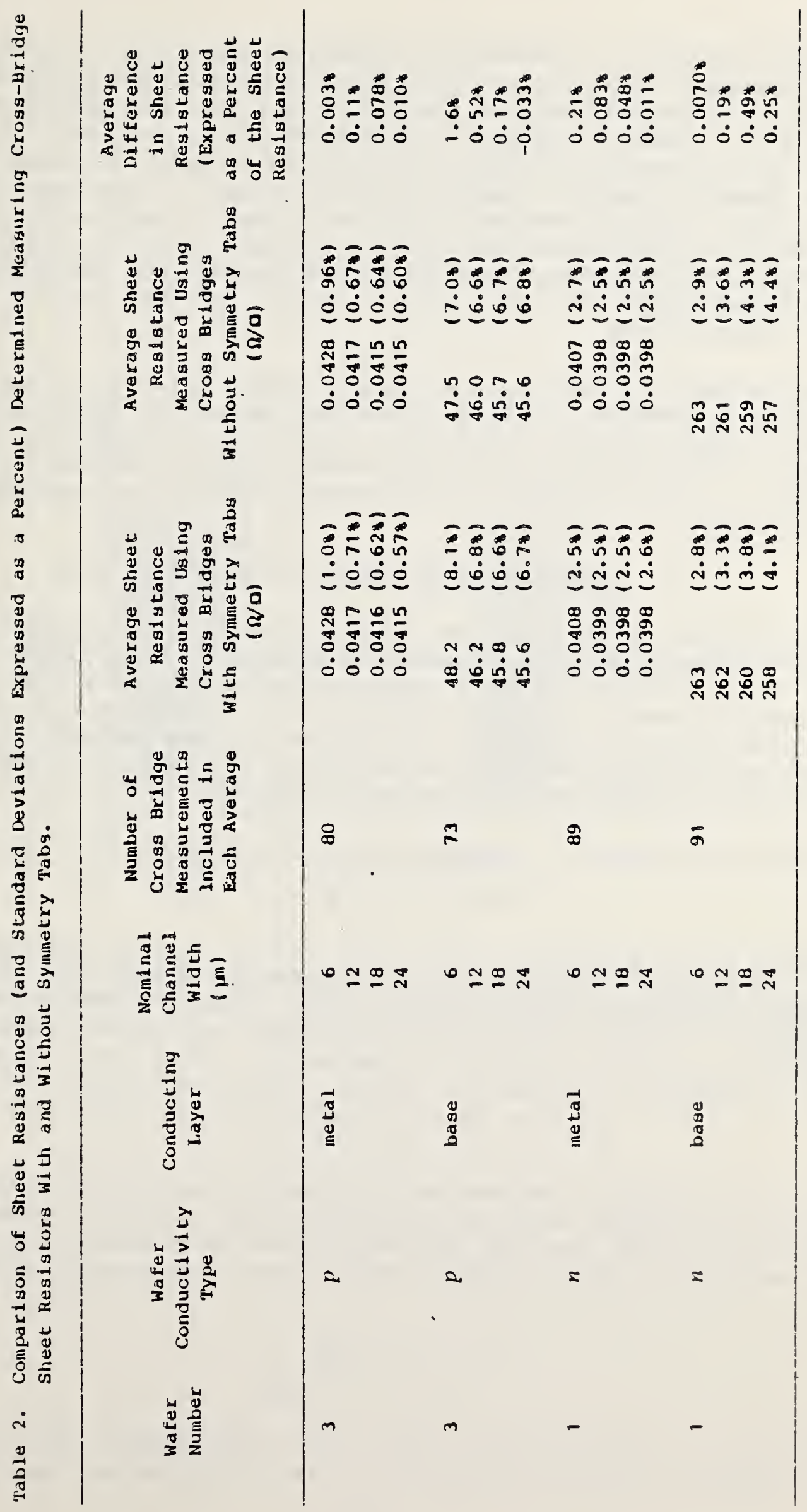


occurring, variations. Furthermore, systematic variations in $R_{S}$ could also cause an average difference in $R_{S}$ between the cross bridges with and without tabs to be measured because the devices are spatially separated. The values of the expected systematic differences in $R_{s^{\prime}}$ obtained by estimating the average incremental systematic resistivity between structures with and without symmetry tabs from inspection of wafer maps of the sheet resistance, are much less than the measured difference values. Based on these considerations and the relative magnitudes of the uncertainties, it can be concluded that within the measurement accuracy of the present data, the differences between cross bridges with and without symmetry tabs are found to be statistically insignificant.

The data in table 2 show that the measured sheet resistance is found to increase with decreasing linewidth. This behavior may be due to nonuniform resistivity profiles and to cross-sectional effects in layers of finite thickness. Edge effects (edge slope in metal, corner rounding in doped layers) become a larger fraction of the nominal width of the structure in narrower structures.

\section{SHUNTING EFEECT CAUSED BY THE VOLTAGE TAPS}

The voltage taps on the conducting channel that forms the bridge partially shunt the current; the channel current penetrates into the taps. At the voltage taps, the width of the conducting channel increases and the resistance per unit length of channel decreases [9]. Therefore, the finite width of the taps reduces the measured voltage and the computed value of the resistance between the taps compared to the voltage and resistance which would be measured if the taps were infinitesimally narrow.

The voltage difference $\Delta \mathrm{V}$ measured between the bridge taps is given by

$$
\Delta V=I\left(R_{1}+R_{2}\right)
$$

where $I$ is the current in the conducting channel and the sum $\left(R_{1}+R_{2}\right)$ is the total resistance between the midlines of the two voltage taps, as shown in figure 4. In terms of the sheet resistance $R_{S^{\prime}}$ the resistance $R_{1}$ is given by

$$
R_{1}=R_{S} \frac{I-M}{W} \text {, }
$$

where $L$ is the distance between the midlines of the two voltage taps, $M$ is the width of the taps, and $W$ is the width of the conducting channel of the bridge. By assuming that the taps are composed of material with the same sheet resistance as the conducting channel, the resistance $R_{2}$ may be written

$$
R_{2}=R_{s} \frac{M}{W+N_{\text {eff }}} \text {, }
$$

where $\mathrm{N}_{\text {eff }}$ is an effective tap length which corresponds to the additional width that the conducting channel would have if the current were uniform in 


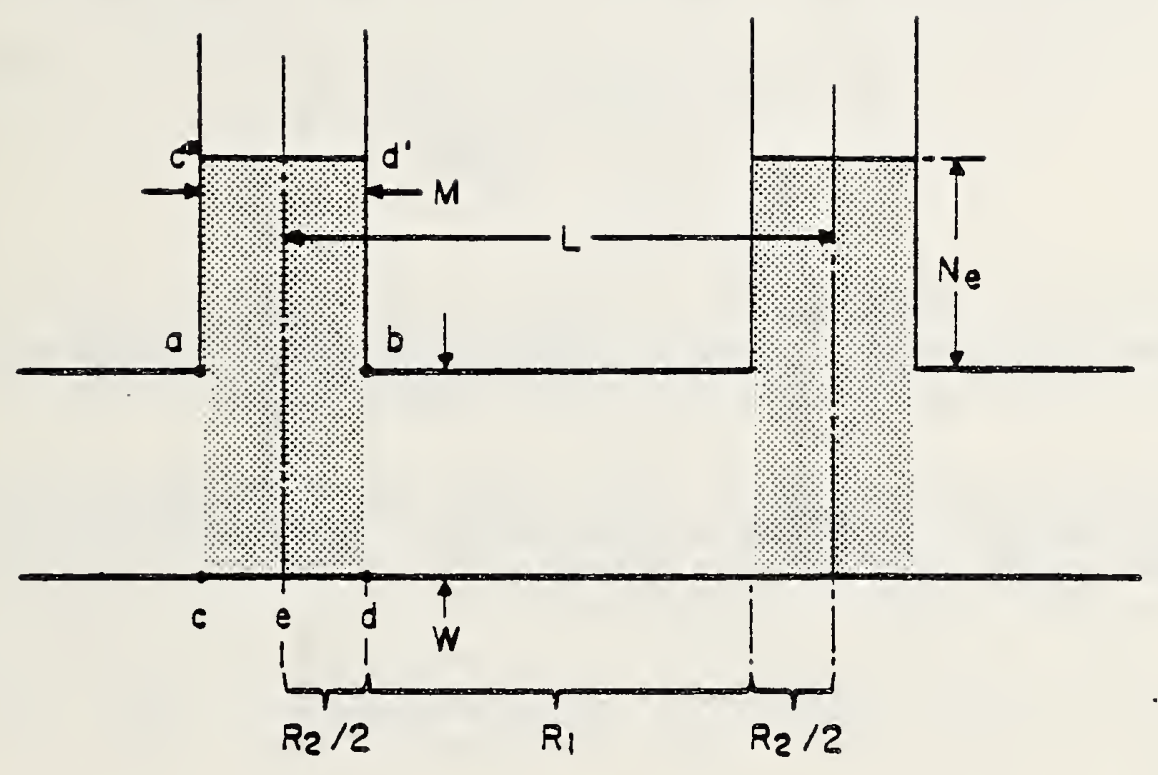

Figure 4. Schematic diagram of the bridge. The resistance of the conducting channel between the voltage taps is $R_{1}$. The resistance of each of the shaded portions of the structure is $R_{2}$. The midlines of the voltage taps are the dash and dotted lines. The geometrical bridge length $I$, tap width $M$, and channel width $w$ are shown. The effective length of the taps is the distance $\mathrm{K}_{e}$. The voltage difference between the taps is considered to be the potential difference between the midlines of the taps. 
the region between the edges of each of the voltage taps, the region between $c^{\prime} c$ and $d \cdot d$ in figure 4.

The actual current density in this region is not uniform. Referring to the figure, progressing upward from point e along the midline of the tap, the current density decreases, starting from the value it has in the conducting channel far away from the taps. The distance the current actually penetrates into each tap is limited only by the end of the tap, although it is affected by the details of the contact window. Nevertheless, the distance $\left(W+N_{e f f}\right)$ can be thought of as the electrically equivalent width of the conducting channel caused by the shunting effect of the voltage taps.

In terms of the geometrical dimensions of the bridge, the measured voltage difference between the taps, given in eq (1), can be written

$$
\Delta V=I_{S}\left[\frac{L-M}{W}+\frac{M}{N_{\text {eff }}+W}\right] \text {, }
$$

or

$$
\Delta V=I R_{S} \frac{L}{W}\left[1-\frac{M}{L}\left(\frac{N_{\text {eff }}}{N_{\text {eff }}+W}\right)\right] .
$$

From eq (5), the shunting caused by the taps can be considered to be a decrease in the electrical length of the bridge. The effective electrical length of the bridge $I_{\text {eff }}$ is then given by

$$
I_{\text {eff }}=L\left[1-\frac{M}{L}\left(\frac{N_{\text {eff }}}{N_{\text {eff }}+W}\right)\right] \text {. }
$$

Defining the quantity $\varepsilon$ by:

$$
\varepsilon=\frac{N_{\text {eff }}}{N_{\text {eff }}+W},
$$

the effective bridge length is

$$
L_{\text {eff }}=L-M \varepsilon \text {. }
$$

The effective bridge length is reduced by the amount $M \varepsilon$.

If $\mathrm{N}_{\text {eff }}$ is zero, $\varepsilon$ is zero and $\mathrm{L}_{\text {eff }}$ equals $\mathrm{L}$; there are no shunting effects and there is no difference between the electrical and geometrical lengths of the bridge. If $\mathrm{N}_{\text {eff }}$ is large compared to $\mathrm{W}, \varepsilon$ is greater than zero but always less than unity. This situation corresponds to each tap acting as a short along one side of the conducting channel. The electrical length of the bridge $L_{\text {eff }}$ is smaller than the geometrical length $L$ by some fraction $\varepsilon$ of the tap width $M$. 
The shunting effect of the voltage taps introduces an error into the width measurement by causing the electrical length of the bridge to be shorter than the physical length. The equation for the measured channel width $w_{\text {meas }}$ is

$$
W_{\text {meas }}=R_{S} L \frac{I}{\Delta V} \text {. }
$$

Substituting for $\Delta V$ from $e q(5)$ and inserting the parameter $\varepsilon_{\text {, }} w_{\text {meas }}$ is given by

$$
W_{\text {meas }}=W\left[\frac{1}{1-\frac{M \varepsilon}{I}}\right] \text {. }
$$

Using the relation between $L$ and $L_{\text {eff' }}$ eq $(7)$, this may be written

$$
\frac{W_{\text {meas }}}{W}=\frac{L}{L_{\text {eff }}} \text {. }
$$

The shunting effect of the voltage taps acts to increase the measured width compared to the actual electrical channel width. (The inverse relationship in eq (10) occurs because of the inverse relationship between $W$ and $L$ in eq (2).)

An actual calculation of $\mathrm{N}_{\text {eff }}$ would provide a value for $\mathrm{M}_{\varepsilon}$ which could be used as a correction factor for $L$, according to eq (8). The maximum allowable physical value for $\varepsilon$ occurs when the voltage taps produce the largest possible shunting effect which is a short circuit along one edge of the conducting channel, between points $a$ and $b$. The average electrical decrease in the conducting channel length would be $M / 2$ in this situation. That is,

$$
L>L_{\text {eff }}>\mathrm{L}-\frac{M}{2}
$$

It is believed that this simple model substantially overestimates the maximum possible systematic inaccuracy in the measured linewidth. It also ignores the expected dependence of $\varepsilon$ on $w$, which is suggested by the definition of $\varepsilon$.

Using methods similar to those used in the analysis of the cross sheet resistor [5], the ratio $\mathrm{M} / \mathrm{N}$ eff was estimated. The technique, which is a numerical iterative approach to the chain rule, involves calculating values for $\mathrm{M} / \mathrm{N}$ eff as a function of the ratios $N / M, L / M$, and $W / M$. Considering $M / N_{e f f}$ to be $a$ function of only one of the ratios while the other two are held constant, it can be established through the iterative process that $M / N_{\text {eff }}$ asymptotically approaches a maximum for small values of $M$ compared with $L$. Specifically, for $M<L L$ and $M<2 W$, it was found that $M / N$ eff approached a value of approximately 6 . 


$$
\varepsilon=\frac{1}{1+\frac{W}{M}\left(\frac{M}{N_{\text {eff }}}\right)} \text {. }
$$

the value of $\varepsilon$ can be estimated to be always less than approximately 0.15 for all cross bridges used in this study.* This estimate agrees with a previous analysis based on conformal transformations [10]. It implies that ( $I-I_{e f f}$ ) is less than $0.9 \mu \mathrm{m}$ and the ratio $\mathrm{w}_{\text {meas }} / \mathrm{W}$ is no greater than 1.005 . Experimental confirmation of this result is unavailable because the linewidth calculated from the structures presented in figure 2 showed no statistically significant variation. The uncertainties and scatter in the data were larger than the expected differences caused by tap shunting. The primary reason for this was filleting at the corners where the taps meet the conducting channels. This commonly occurs with wet chemistry etching and thermal diffusions. Visual observation indicates that some of the narrower taps are more than twice their design width at the point where they meet the conducting channel of the bridge. The filleting is not uniform and some of the taps are not symmetrical. The result is that the parameter $M$ is not well defined and the variability contributes to the uncertainty in $W_{\text {meas }}$.

It is important that the tap shunting effect causes a systematic overmeasurement of the linewidth. The shunting does not affect the resolution of the measurement or the utility of cross bridges provided that process-induced variations in structure geometry or sheet resistance do not cause large measurement variations. Such effects do not appear to influence cross bridges with the dimensions used in this study for linewidth uniformity measurements, but may become important when the linewidth of the structures is in the range of a micrometer or less.

\section{TAP-TO-CORNER DISTANCE STUDY}

The tap-to-corner distance study test structures are a series of bridge sheet resistors that have one of the two voltage taps placed at different distances from one end of the conducting channel which forms the bridge, as shown in figure 3. Each structure is a bridge sheet resistor, formed in the metal or base layers, with a 20-Im wide conducting channel and 6-Im wide voltage taps.

The tap-to-corner distance structures were intended to study the effect of the proximity of a bridge tap to the current contact corner. The corner represents the end of the linear portion of the bridge. It is expected that the current density is nonuniform in the region of the current contacts and that this nonuniformity affects the measured value of the potential between the voltage taps. In all potentiometric measurements of this type, uniform current density in the vicinity of the taps is a necessity.

The voltage tap-to-corner distance between the bottom of the bridge and the lower voltage tap varies from structure to structure in the sequence 0, 20, 40, 60, and $80 \mu \mathrm{m}$. As can be seen in the diagrams, the distance is measured

* For the cross bridges used in this study, $M$ was always less than or equal to $W$. For the case $M$ equals $W, M / N_{\text {eff }}$ was estimated to be 5.6 . 
from the midline of the tap to the upper edge of the conducting path connecting the bridge to the probe pad at the lower left.

Because the structures were designed with constant length of the linear portion and fixed upper tap position, the distance between the two voltage taps also changes as the lower tap-to-corner distance changes. The length of the bridge changes in the sequence 160,140,120,100, and $80 \mathrm{~mm}$. The shunting effect caused by the width of the voltage taps is an interference to the measurement, and compensation must be made for this effect.

The data obtained using the tap-to-corner distance test structures are presented in table 3. The calculated channel widths, averaged over about 60 devices of each type on each wafer, are approximately 23 im for the base structures and $17 \mu \mathrm{m}$ for the metal structures. The difference between these values and the design value of $20 \mathrm{~m}$ is due to lateral diffusion of the base layer, overetching of the oxide cut for the base layer diffusion, and overetching of the metal layer.

To evaluate these linewidth results, account has to be taken of the shunting effect due to the voltage taps; a value for $\varepsilon$ must be assumed. The best value for $\varepsilon$ is taken as 0.15 , as described in the previous section. Using this value, Ieff is calculated from eq (8). Then, in the rightmost column, values for $w$ were determined using eq $(10)$. The measurements on devices with the tap-to-corner distances equal to zero yield a significantly larger value of linewidth than the other devices in each case. Typically, the bridges with the tap-to-corner distance greater than zero agree to within a few tenths of a percent, whereas the bridge with zero tap-to-corner distance yields a corrected linewidth which is about 1 percent higher than the mean of the other values.

Generally, the results suggest that when the tapwidth is much smaller than the channel width, the voltage tap near the end of the conducting channel should be placed at least one channel width (nominally 20 m in this case) away from the end of the straight portion of the conducting channel which forms the bridge. Because the cross bridge can easily be designed so that the tap-to-corner distance is at least as large as the channel width, a detailed statistical analysis in support of this empirical study is not felt to be necessary.

\section{SUMMARY AND DESIGN CONSIDERATIONS}

For cross-bridge sheet resistor test structures with nominally 6-, 12-, 18-, and 24-um wide conducting channels, three geometrical factors affecting the accuracy of the measurement of linewidth have been addressed. The three factors are the presence or absence of symmetry tabs on three arms of the cross portion of the structure, the finite width of the bridge voltage taps, and the distance between a voltage tap and the end of the linear portion of the bridge conducting channel ("tap-to-corner" distance). In each case the effects were found to be less than about 1 percent on the measurement of linewidth for cross-bridge structures with the dimensions used in this study.

A characteristic of these results was that in every case the standard deviation of the mean of the measurements was always larger than the expected 


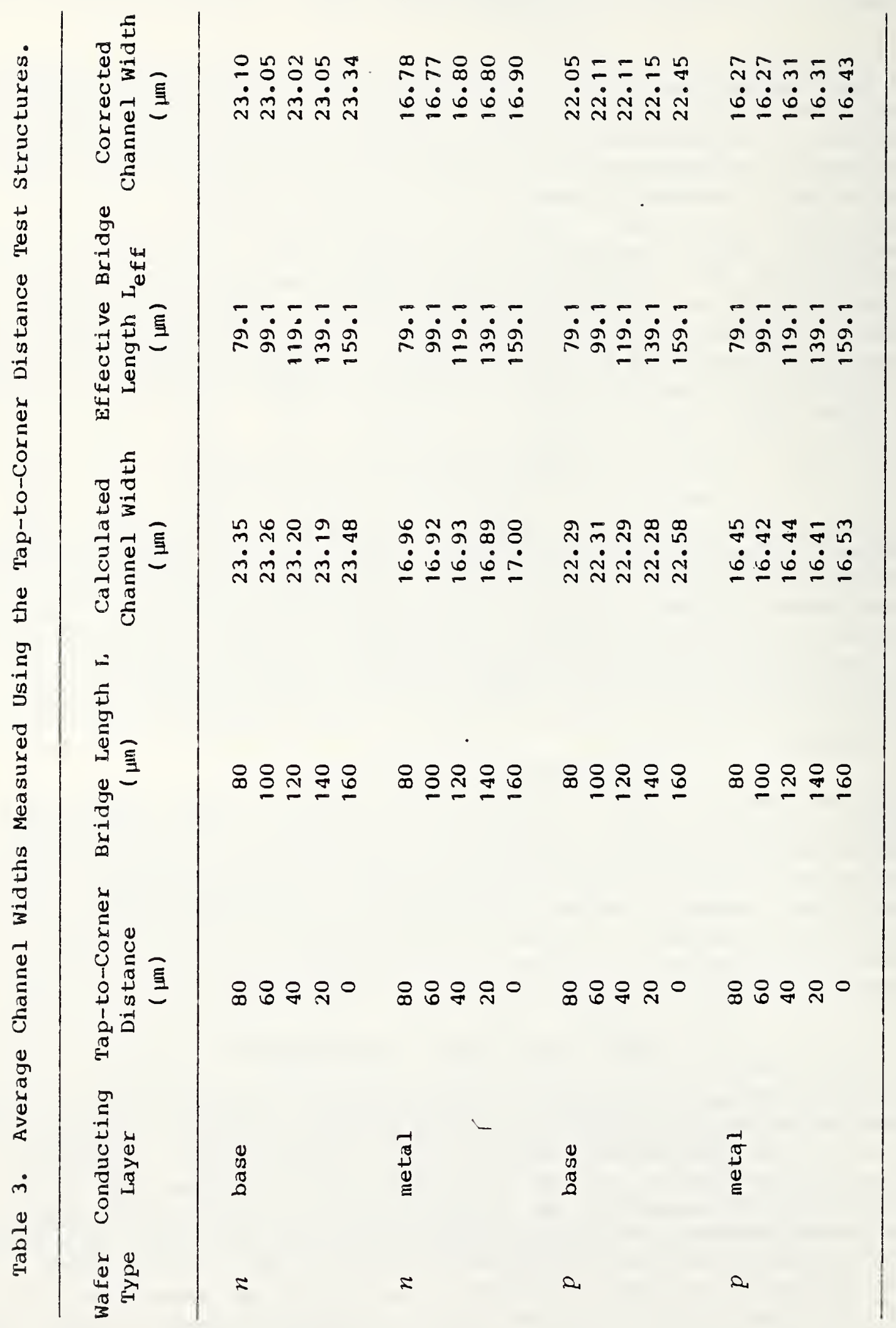


uncertainty due to the instruments. This outcome is not surprising because the results were averaged across the wafers and therefore include the systematic process-induced intrawafer variations in sheet resistance and linewidth. (This situation was discussed in sec. 3.) Furthermore, there are random variations caused by the fabrication process which contribute to the measurement variations. Random variations occurring on the scale of the dimensions of the structures include edge roughness, corner rounding, pinholes, and other imperfections, including those present on the mask set. Averaging over a wafer reduces the random variations (if they are distributed in a truly random fashion), but usually increases the statistical uncertainty due to process variations which vary slowly across a wafer, the systematic variations.

With the process-related systematic variations of the wafers used in this study, it was not possible to measure reliably differences due to the geometrical factors addressed here of less than 1 percent. Each of the geometrical factors included in this study was found to have an effect of less than 1 percent on the measurement of sheet resistance and linewidth, with the exception of the case where the tap-to-corner distance was 0 .

It is important to differentiate between factors which affect the accuracy of a measurement and those which affect the resolution of the measurement. The absolute accuracy of the results is governed by the experimental apparatus, the geometrical design of the cross bridge, process uniformity, and the measurement strategy. The geometrical factors addressed in this study introduce systematic errors in the sheet resistance and linewidth measurements. process-induced random variations and systematic process-related variations contribute to the imprecision of the measurement. If the fabrication process is under control and if the random variations are small (or if enough measured values are averaged), the cross-bridge sheet resistor can be used to obtain extremely precise results.

Based upon the results reported in this paper, geometrical factors which lead to inaccuracy in the measurements on cross-bridge sheet resistors are: systematic in that they affect all structures equally; (2) quantifiable, because the magnitude of the effect, or at least the maximum effect, on the measurement can be determined; and (3) minimizable, through proper design and use of the test structure.

A test structure that is well designed and well characterized is one that can be used to obtain unambiguous results that are not affected by other materials or process characteristics. The information needed to develop a welldesigned test structure is obtained by careful modeling of the structure and through comparison with measurements on actual structures. This study is part of the process toward completing the development of the cross-bridge sheet resistor test structure. With the information obtained from these results, details of the suggested design for the cross-bridge sheet resistor are further resolved. The suggested design conforms with the objectives of the modular probe-pad array $[4,11]$. An outline drawing of the structure is shown in figure 5, which also identifies the various geometrical parameters. 
If the intent is to avoid having to make corrections to the data and if systematic inaccuracy caused by geometry is acceptable providing it is less than about 1 percent, then the following inequalities should be met:

$$
\begin{aligned}
& \mathrm{N}>\mathrm{M} \\
& \mathrm{L}>15 \mathrm{M} \\
& \mathrm{W} \geq \mathrm{M} \\
& \mathrm{A}_{1}>\mathrm{W} \\
& \mathrm{A}_{\mathrm{O}}>\mathrm{W}
\end{aligned}
$$

Based on the present data, the proposed design results in geometry-related inaccuracies significantly below the 1-percent level. Nevertheless, it is possible that, in some processes, variations in edge and sidewall quality, materials irregularities, variations in etching, and other process-related, randomly occurring imperfections will contribute to inaccuracy levels greater than 1 percent. Even in that case, however, experimental data from the cross bridge and from other test structures, along with appropriate models, can be used to evaluate and possibly compensate for effects of nonideal processing.

\section{Acknowledgments}

The authors are indebted to Schuyler D. Grant for the calculations of the ratio $M / N_{e f f}$, to $W$. Murray Bullis for a thorough review of the manuscript, and to $E$. Jane Walters for editorial preparation of the manuscript. 


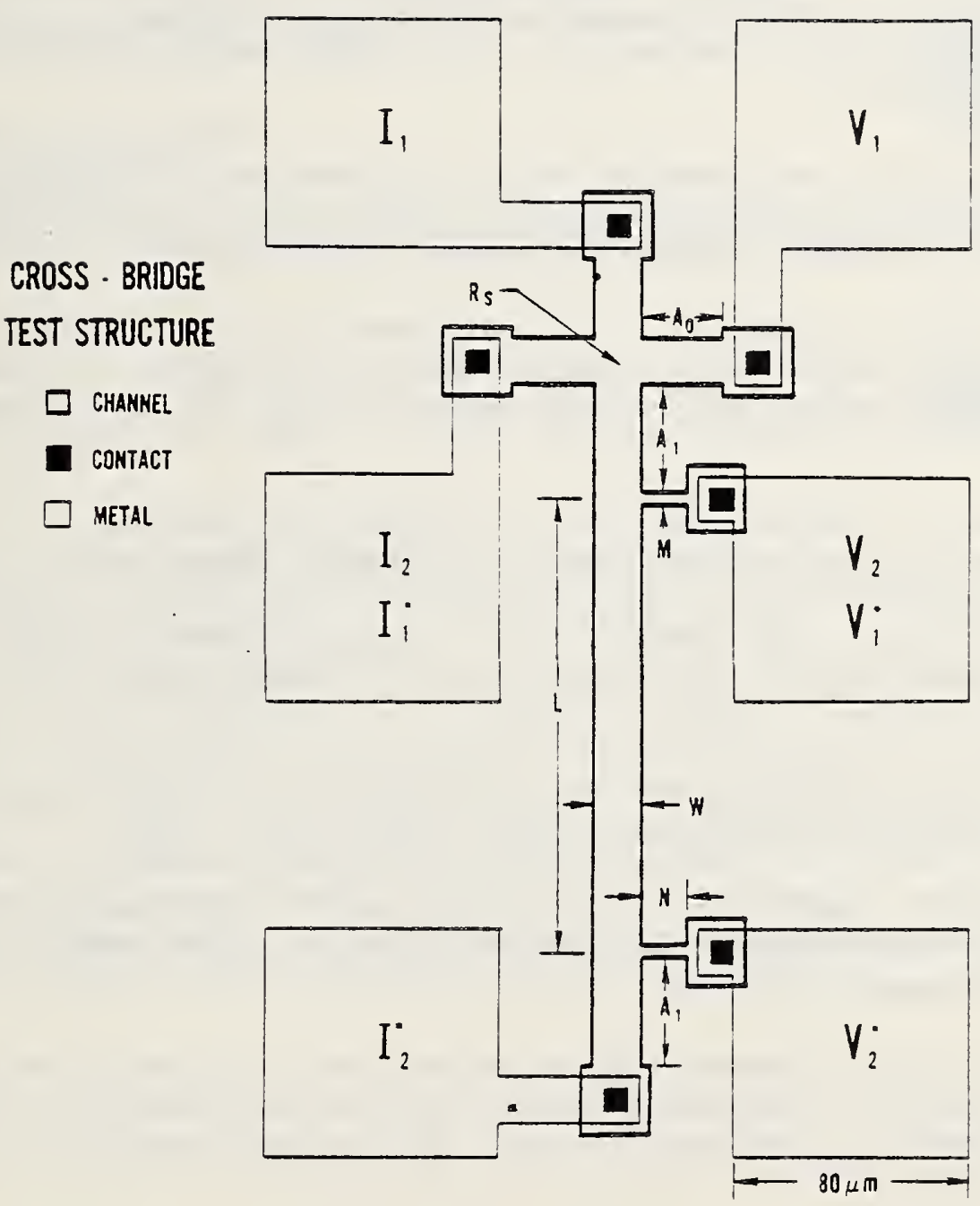

Figure 5. Outline drawing of a cross-bridge sheet resistor test structure. The sheet resistance $R_{S}$ and the effective Iinewidth $w$ are determined by $a$ van der Pauw and a bridge measurement. Reference [1] describes the measurement technique. The geometrical parameters identified in the figure are described in the text. 
1. Buehler, M. G., Grant, S. D., and Thurber, W. R., Bridge and van der Pauw Sheet Resistors for Characterizing the Linewidth of Conducting Layers, J. Electrochem. Soc. 125, 650-654 (1978).

2. Russe11, T. J., Maxwe 11, D. B., Reimann, C. T., and Buehler, M. G., A Microelectronic Test Pattern for Measuring Uniformity of an Integrated Circuit Fabrication Technology, Solid State Technology 22 (2), 71-74 (February 1979).

3. Linholm, L. W., and Buehler, M. G., A Cross-Bridge Structure Array for Characterizing Intrachip Linewidth Variations, Electrochemical Society Extended Abstracts, Abstract No. 191, May 1979.

4. Carver, G. P., Linholm, L. W., and Russell, T. J., The Use of Microelectronic Test Structures to Characterize IC Materials, Processes, and Processing Equipment, Solid State Technology 23 (9), 85-92 (September 1980).

5. David, J. M., and Buehler, M. G., A Numerical Analysis of Various Cross Sheet Resistor Test Structures, Solid-State EZectron. 20, 539-543 (1977).

6. Buehler, M. G., and Thurber, W. R., An Experimental Study of Various Cross Sheet Resistor Test Structures, J. Electrochem. Soc. 125, 645-650 $(1978)$.

7. Carver, G. P., Mattis, R. L., and Buehler, M. G., Microelectronic Test Patterns NBS-12 and NBS-24, NBSIR 81-2234 (May 1981).

8. Leedy, T. F., and Liu, Y. M., Semiconductor Measurement Technology: Microelectronic Processing Laboratory at NBS, NBS Special Publication 400-53 (December 1978).

9. Buehler, M. G., Cross-Bridge Sheet Resistor, in Semiconductor Measurement Technology Progress Report, July 1 to September 30, 1976, W. M. Bullis and J. F. Mayo-Wells, eds., NBS Special Publication 400-36, pp. 45-46 (July 1978).

10. Hall, P. M., Resistance Calculations for Thin Film Patterns, Thin Solid Films 1, 277-295 (1968).

11. Buehler, M. G., Comprehensive Test Patterns with Modular Test Structures: The 2 by $N$ Probe-Pad Array Approach, Solid State Technology $22(10), 89-94$ (October 1979). 
MBS IILA IREV. O-78)

\begin{tabular}{|c|c|}
\hline $\begin{array}{c}\text { U.S. DEPT. OF COMM. } \\
\text { BIBLIOGRAPHIC DATA } \\
\text { SHEET }\end{array}$ & 1. PUBLICATION OR REPORT NO. \\
\hline
\end{tabular}

4. TITLE AND SUBTITLE

Design Considerations for the Cross-Bridge Sheet Resistor
7. AUTHOR(S)

G. P. Carver, R. I. Mattis, and M. G. Buehler
9. PERFORMING ORGANIZATION NAME AND ADDRESS

NATIONAL BUREAU OF STANDARDS

DEPARTMENT OF COMMERCE

WASHINGTON, DC 20234

12. SPONSORING ORGANIZATION NAME AND COMPLETE ADORESS (Stroet, CIty, state, 2IP)

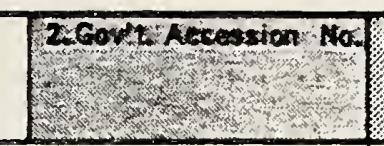

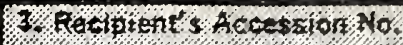

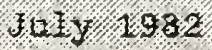

5. Publication Date July 1982

6 Pertonning Organization Code.

8. Performing Organ. Report No.

Document describes a computer program; SF-185, FIPS Software Summary, is attached.

16. ABSTRACT (A 200-word of loss factual summary of most significant information. If document includes a significant bibliography or literature survoy, mention it hore.)

The cross-bridge sheet resistor test structure is used to obtain the sheet resistance and electrical linewidth of a conducting layer. It has been used to characterize various conducting layers found in an integrated circuit fabrication process and to evaluate lithographic equipment used for processing photomasks and wafers. Three geometrical design factors for the cross bridge have been investigated and are shown to cause systematic inaccuracies of less than one percent in the sheet resistance and linewidth measurements. Based upon experimental results from sequences of devices with incrementally different geometrical parameters, several design criteria for the cross-bridge sheet resistor have been established.

17. KEY WORDS (six to twelve entriea; alphabetical order; capitallze only the first letter of the first key word unlese a proper namp; seperated by semicolons)

Cross-bridge structure; linewidth; microelectronic test structure; process control; sheet resistance; test structure.

18. AVAILABILITY Xnlimited

For Official Distribution. Do Not Release to NTIS

Order From Sup. of Doc., U.S. Government Printing Office, Wasinington, DC 20402, SD Stock No. SNOOO3-003-

X. Order From National Technical Information Service (NTIS), Springfield, VA. 22161

\begin{tabular}{|l|c|}
\hline $\begin{array}{l}\text { 19. SECURITY CLASS } \\
\text { (THIS REPORT) }\end{array}$ & $\begin{array}{c}\text { 21. NO. OF } \\
\text { PRINTED PAGES } \\
\text { UNCLASSIFIED }\end{array}$ \\
\hline $\begin{array}{l}\text { 20. SECURITY CLASS } \\
\text { (THIS PAGE) }\end{array}$ & 21 \\
UNCLASSIFIED & \$0. PTICE \\
\hline
\end{tabular}




